\section{Diagnostic value of supersonic shear impulse elastography for malignant cervical lymph nodes: a Bayesian analysis}

\author{
Yuxuan Qiu ${ }^{1,2 *}$, Zhichao Xing ${ }^{2 *}$, Qianru Yang ${ }^{1}$, Yan Luo ${ }^{1}$ \\ 'Department of Ultrasound, Sichuan University West China Hospital, Chengdu, ${ }^{2}$ Center of \\ Thyroid \& Parathyroid Surgery, Sichuan University West China Hospital, Chengdu, China
}

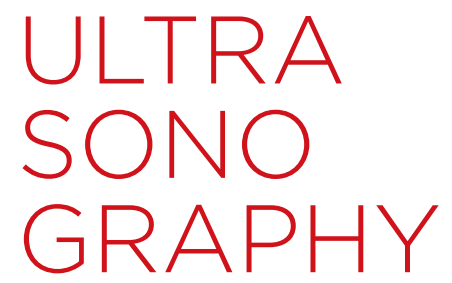

META-ANALYSIS

https://doi.org/10.14366/usg.21107 pISSN: 2288-5919 - elSSN: 2288-5943 Ultrasonography 2022;41:279-290

Received: May 14, 2021

Revised: July 30, 2021

Accepted: July 31, 2021

Correspondence to:

Yan Luo, MD, Department of

Ultrasound, Sichuan University West

China Hospital, No. 37 Guo Xue Xiang, Chengdu 610041, China

Tel. +86-028-85422303

Fax. +86-028-85422303

E-mail: luoyan@wchscu.cn

*These authors contributed equally to this work.

This is an Open Access article distributed under the terms of the Creative Commons Attribution NonCommercial License (http://creativecommons.org/ licenses/by-nc/4.0/) which permits unrestricted noncommercial use, distribution, and reproduction in any medium, provided the original work is properly cited.

Copyright (C) 2022 Korean Society of Ultrasound in Medicine (KSUM)

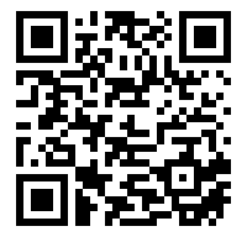

How to cite this article:

Qiu Y, Xing Z, Yang Q, Luo Y. Diagnostic value of supersonic shear impulse elastography for malignant cervical lymph nodes: a Bayesian analysis. Ultrasonography. 2022 Apr;41(2):279-290. 
in terms of whether they are malignant or benign. Cervical lymph node assessment can predict the prognosis and contribute to the management plans of patients with primary tumors in other places [1]. Conventional ultrasonography (US) is the first choice of imaging modality for diagnosing malignant cervical lymph nodes preoperatively. It can characterize the distribution and morphology of superficial lymph nodes, including their shape, border, internal structure, and vascularity [2]. However, there are no standardized criteria for US in the diagnosis of malignant lymph nodes with satisfactory sensitivity and specificity $[3,4]$.

In recent years, US elastography has been introduced to measure the elasticity of lymph nodes, which is a parameter based on the strain or deformation in response to a physical force related to the degree of malignancy, as most malignancies reliably exhibit reduced elasticity and increased stiffness due to the nature of neoplastic growth [5]. Elastography can be divided into quasi-static elastography and shear wave elastography, including supersonic shear impulse (SSI) elastography and acoustic radiation force impulse (ARFI) elastography. Quasi-static elastography involves collecting numerical data with a quasi-static method, such as the elasticity score, which is a qualitative classification, and the strain index or the muscle-to-lymph node strain ratio, which are often considered as semiquantitative; this technique is widespread and has been extensively studied. A meta-analysis of quasistatic elastography demonstrated that the summary sensitivity and specificity for diagnosing superficial malignant lymph nodes were 0.74 and 0.90 for the elasticity score and 0.88 and 0.81 for the strain ratio, respectively [1]. SSI elastography is an objective and quantitative modality, which is less operator-dependent. SSI elastography analyzes tissue stiffness using the elastic modulus ( $\mathrm{KPa}$ ), which provides absolute quantification of lymph node elasticity [6], while ARFI uses the shear wave speed $(\mathrm{m} / \mathrm{s})$ for the quantitative analysis. In addition, SSI elastography does not require freehand compression, making it independent of the compression technique [6].

A previous systematic review reported that the sensitivity and specificity of shear wave elastography in the diagnosis of cervical lymph nodes were 0.81 and 0.85 , respectively [3]. However, that study used the maximum elastic modulus for SSI and shear wave velocity (m/s) for ARFI as a cutoff value, and did not systematically provide results for the maximum, mean, minimum, and standard deviation (SD) values of the elastic modulus. Furthermore, studies of SSI elastography for evaluating cervical lymph nodes have been carried out in small and selected patient samples, and have had observational designs. Therefore, this study was conducted to provide new clinical evidence regarding differences in elastic modulus values using SSI elastography between malignant and benign cervical lymph nodes and the diagnostic performance of SSI elastography for diagnosing malignant cervical lymph nodes.

\section{Materials and Methods}

This systematic review and meta-analysis of diagnostic test accuracy was conducted following the Preferred Reporting Items for Systematic Review and Meta-Analyses (PRISMA) extension for diagnostic test accuracy statement [7]. This systematic review and meta-analysis was registered with PROSPERO (https://www.crd.york. ac.uk/prospero/) with an ID of CRD42020165574.

\section{Literature Search}

A systematic search was conducted in PubMed, Embase, and Cochrane Library Central Register of Controlled Trials databases up to December 1, 2020. The search strategy employed Boolean logic to merge discrepant concepts and synonyms. The search terms were the following: (cervical lymph node OR neck lymph node OR cervical lymphadenopathy $O R$ neck node $O R$ cervical node) AND (elastography OR sonoelastography OR sonoelastography OR supersonic imaging OR supersonic shear imaging OR supersonic shear wave elastography OR supersonic shear-wave elastography). Two independent investigators first screened the title and abstract of each article. The references of the eligible articles and reviews were then screened.

\section{Eligibility Criteria}

First, English studies or their subsets that reported SSI elastography data as a predictor of malignant cervical lymph nodes, were eligible for inclusion. Then, the final diagnoses of cervical lymph nodes were determined based on histopathology reports after surgery or fineneedle aspiration biopsy. The exclusion criteria were as follows: (1) articles that did not fall within the scope of this review; (2) review articles, editorials or letters, comments, and conference proceedings; (3) case reports or case series.

\section{Data Extraction}

Descriptive data were extracted by one investigator and then confirmed by another researcher. The extracted descriptive data included the study characteristics (authors, year of publication, region, study design, duration of patient recruitment, and sample size), clinical characteristics (the number of lymph nodes, both benign and malignant, patient age, type of primary tumor, elastic modulus [ $\mathrm{kPa}$ ] and cutoff values), and diagnostic accuracy test characteristics. Two reviewers independently extracted the numerical data. Inconsistent extractions were resolved by consensus. When the data were not extractable, an attempt was made to contact the corresponding authors for additional data. 


\section{Quality Assessment}

Two reviewers independently assessed the risk of bias and concerns about applicability based on the Quality Assessment of Diagnostic Accuracy Studies 2 (QUADAS-2) tool [8]. Discrepancies were resolved by consensus.

\section{Data Synthesis}

For all mean, maximum, minimum, and SD values of the elastic modulus, the mean difference (MD) with a $95 \%$ confidence interval $(\mathrm{Cl})$ was analyzed. Heterogeneity was examined using the Q-test and the $I^{2}$ statistic. When studies were heterogeneous $(P<0.1$ or $\left.I^{2}>50 \%\right)$, a random effects model was used; otherwise, a fixed effects model was applied [9].

For diagnostic characteristics, a Bayesian bivariate model was implemented using integrated nested Laplace approximation (INLA) [10]. Accurate posterior marginal distributions for sensitivity and specificity, as well as all hyperparameters and covariates, were directly obtained by a bivariate model with no need for conventional Markov-chain Monte Carlo sampling [11,12]. Further, univariate estimates of sensitivity and specificity with $95 \%$ credible intervals (Crls), as well as the summary receiver operating characteristic (SROC) curve, were directly available for interpretation. Moreover, the area under the receiver operating characteristic curve (AUC) values with $95 \% \mathrm{Crls}$ were calculated. The summary positive and negative likelihood ratios ( $L R+$ and $L R-$, respectively) were calculated from the summary sensitivity and specificity estimates, and the same procedure was carried out for diagnostic odds ratios
(ORs) and risk difference (RD). Alternative models were compared using the deviance information criterion (DIC), with a lower DIC considered to be better. In model 1, the sensitivity and specificity were modeled in the bivariate model. Models 2, 3, and 4 indicate that the sensitivity and false-negative rate (1-specificity), falsepositive rate (1-sensitivity) and specificity, false-positive rate (1-sensitivity) and false-negative rate (1-specificity) were modeled in the bivariate model, respectively. The threshold effect was tested using Spearman correlations, and a P-value less than 0.05 was considered to indicate a significant threshold effect. Funnel plot asymmetry was also examined to enable a valid assessment of the extent and impact of publication and selective reporting bias in studies of diagnostic accuracy. Subgroup analyses were performed according to the study design (prospective or retrospective), age of included patients (over or less than the median age), histopathology (papillary thyroid cancer or other carcinoma), and prevalence of malignancy (over or less than the median rate).

All analyses were conducted using R software version 4.0.4 (R Foundation for Statistical Computing, Vienna, Austria; https://www. r-project.org) with the R package meta (4.18-2), meta4diag (2.0.8), and INLA (21.02.23) with their required packages.

\section{Results}

\section{Literature Search and Study Characteristics}

After excluding duplicate results and abstract screening, the full texts of potentially eligible publications were reviewed. After

\section{Records identified through database searching 101 PubMed 74 Embase 3 Cochrane}

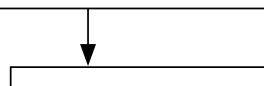

102 Records after duplicates removed
2 Additional records identified through other sources

\begin{tabular}{|c|}
\hline $\begin{array}{c}37 \text { Full-text articles } \\
\text { assessed for eligibility }\end{array}$ \\
\hline $\begin{array}{c}10 \text { Studies included in } \\
\text { qualitative synthesis }\end{array}$ \\
\hline $\begin{array}{c}10 \text { Studies included in } \\
\text { quantitative synthesis } \\
\text { (meta-analysis) }\end{array}$ \\
\hline
\end{tabular}

e-ultrasonography.org

Ultrasonography 41(2), April 2022

Fig. 1. PRISMA diagram of study selection. SSI, supersonic shear imaging.
27 Full-text articles excluded with reasons

3 Reviews

2 Case series

19 Not SSI elastography 3 Diagnostic characteristics unavailable 
applying the exclusion criteria, 10 studies were included in this systematic review. The study selection process is described in Fig. 1.

Ten eligible articles with 590 patients (333 women and 257 men) were included in the study [13-22]. Seven of them were designed prospectively, and three had retrospective designs. Of 892 cervical lymph nodes, 301 (33.7\%) were malignant. The prevalence of malignancy ranged from $19.5 \%$ to $60.7 \%$, with a median of $29.7 \%$. SSI elastography data included mean, maximum, minimum, and SD

Table 1. Characteristics of the included studies

\begin{tabular}{|c|c|c|c|c|c|c|c|c|}
\hline Study & Region & Design & Period & $\begin{array}{l}\text { No. of } \\
\text { patients } \\
\text { (female/ } \\
\text { male) }\end{array}$ & $\begin{array}{l}\text { Age } \\
\text { (range, } \\
\text { year) }\end{array}$ & $\begin{array}{l}\text { Prevalence (\%) } \\
\text { of malignant } \\
\text { lymph nodes } \\
\text { (malignant/total) }\end{array}$ & Primary tumors (no. of lymph nodes) & $\begin{array}{l}\text { Cutoff value } \\
\text { of SSI index } \\
\qquad(\mathrm{kPa})\end{array}$ \\
\hline $\begin{array}{l}\text { Lo et al. } \\
\text { (2019) [13] }\end{array}$ & Taiwan & Prospective & $\begin{array}{l}2015 \text { Aug- } \\
2016 \text { Jan }\end{array}$ & $\begin{array}{c}109 \\
(55 / 54)\end{array}$ & $\begin{array}{c}46 \\
(21-86)\end{array}$ & $\begin{array}{c}22.0 \\
(24 / 109)\end{array}$ & $\begin{array}{l}\text { Oral cancer (6), nasopharyngeal cancer (3), } \\
\text { oropharyngeal cancer (2), hypopharyngeal } \\
\text { cancer (2), lymphomas (2), endometrial } \\
\text { cancer (2), melanoma (1), laryngeal cancer } \\
\text { (1), esophageal cancer (1), lung cancer (1), } \\
\text { prostate cancer (1), unknown primary cancer } \\
\text { (2) }\end{array}$ & Mean: 42 \\
\hline $\begin{array}{l}\text { Kang et al. } \\
\text { (2019) [14] }\end{array}$ & Korea & Retrospective & $\begin{array}{l}2016 \mathrm{Mar}- \\
2018 \mathrm{Jan}\end{array}$ & $\begin{array}{c}127 \\
(80 / 47)\end{array}$ & $\begin{array}{c}50.4 \\
(16-87)\end{array}$ & $\begin{array}{c}31.5 \\
(41 / 130)\end{array}$ & $\begin{array}{l}\text { Papillary thyroid cancer (14), } \\
\text { lymphoproliferative malignancy (6), squamous } \\
\text { cell carcinoma (9), others (2) }\end{array}$ & Max: 54 \\
\hline $\begin{array}{l}\text { You et al. } \\
\text { (2018) [15] }\end{array}$ & China & Prospective & $\begin{array}{l}2014 \text { Aug- } \\
2014 \text { Nov }\end{array}$ & $\begin{array}{c}39 \\
(27 / 12)\end{array}$ & $\begin{array}{c}45.6 \\
(15-67)\end{array}$ & $\begin{array}{c}24.8 \\
(35 / 141)\end{array}$ & Papillary thyroid cancer (35) & $\begin{array}{l}\text { Mean: } 22.1 \\
\text { Max: } 40.2 \\
\text { Min: } 12.4 \\
\text { SD: } 4.1\end{array}$ \\
\hline $\begin{array}{l}\text { Kim et al. } \\
\text { (2018) [16] }\end{array}$ & Korea & Retrospective & $\begin{array}{l}2016 \text { Jan- } \\
2017 \text { Sep }\end{array}$ & $\begin{array}{c}43 \\
(31 / 12)\end{array}$ & $\begin{array}{c}52.4 \\
(35-65)\end{array}$ & $\begin{array}{c}27.9 \\
(12 / 43)\end{array}$ & Papillary thyroid cancer (12) & $\begin{array}{l}\text { Mean: } 23 \\
\text { Max: } 37.5 \\
\text { Min: } 11.7 \\
\text { SD: } 6.9\end{array}$ \\
\hline $\begin{array}{l}\text { Chang et al. } \\
\text { (2018) [17] }\end{array}$ & China & Prospective & $\begin{array}{l}2017 \text { Apr- } \\
2017 \text { May }\end{array}$ & $\begin{array}{c}22 \\
(17 / 5)\end{array}$ & $\begin{array}{c}45.6 \\
(25-70)\end{array}$ & $\begin{array}{c}19.5 \\
(17 / 87)\end{array}$ & Papillary thyroid cancer (17) & $\begin{array}{l}\text { Max: } 29.2 \\
\text { SD: } 3.45\end{array}$ \\
\hline $\begin{array}{l}\text { Chen et al. } \\
\text { (2018) [18] }\end{array}$ & China & Prospective & $\begin{array}{l}2014 \text { Jun- } \\
2016 \text { Mar }\end{array}$ & $\begin{array}{c}62 \\
(15 / 47)\end{array}$ & $\begin{array}{c}43.5 \\
(19-66)\end{array}$ & $\begin{array}{c}22.8 \\
(26 / 114)\end{array}$ & Nasopharyngeal carcinoma (26) & $\begin{array}{l}\text { Mean: } 18.4 \\
\text { Max: } 20.6 \\
\text { Min: } 15.5\end{array}$ \\
\hline $\begin{array}{l}\text { Desmots et } \\
\text { al. (2016) } \\
{[19]}\end{array}$ & France & Prospective & $\begin{array}{c}2010 \mathrm{Apr}- \\
2013 \mathrm{Oct}\end{array}$ & $\begin{array}{c}56 \\
(25 / 31)\end{array}$ & $\begin{array}{c}49 \\
(25-84)\end{array}$ & $\begin{array}{c}48.4 \\
(30 / 62)\end{array}$ & $\begin{array}{l}\text { Squamous cell carcinoma (16), papillary } \\
\text { thyroid carcinoma (4), non-keratinizing } \\
\text { nasopharyngeal carcinoma (1), } \\
\text { mucoepidermoid carcinoma (1), synovial } \\
\text { sarcoma (1), clear cell renal carcinoma (2), } \\
\text { angioimmunoblastic T-cell lymphoma (1), } \\
\text { diffuse large B-cell lymphoma (1), spindle } \\
\text { cell sarcoma (1), melanoma (1), malignant } \\
\text { paraganglioma (1) }\end{array}$ & Max: 31 \\
\hline $\begin{array}{l}\text { Jung et al. } \\
\text { (2015) [20] }\end{array}$ & Korea & Retrospective & $\begin{array}{l}2011 \text { May- } \\
2012 \text { Dec }\end{array}$ & $\begin{array}{c}66 \\
(50 / 16)\end{array}$ & $\begin{array}{l}45.2 \\
\text { (na) }\end{array}$ & $\begin{array}{c}60.7 \\
(51 / 84)\end{array}$ & Papillary thyroid cancer (51) & $\begin{array}{l}\text { Mean: } 29 \\
\text { Max: } 57 \\
\text { Min: } 24\end{array}$ \\
\hline $\begin{array}{l}\text { Choi et al. } \\
\text { (2013) [21] }\end{array}$ & Korea & Prospective & $\begin{array}{l}2010 \text { Oct- } \\
2010 \text { Oct }\end{array}$ & $\begin{array}{c}15 \\
(7 / 8)\end{array}$ & $\begin{array}{c}54.2 \\
(38-73)\end{array}$ & $\begin{array}{c}50.7 \\
(34 / 67)\end{array}$ & $\begin{array}{l}\text { Papillary thyroid carcinoma (10), salivary duct } \\
\text { carcinoma (2), ex pleomorphic adenoma of } \\
\text { the submandibular gland (1), melanoma of } \\
\text { the scalp (1), squamous cell carcinoma of the } \\
\text { hypopharynx (1) }\end{array}$ & Max: 19.44 \\
\hline $\begin{array}{l}\text { Bhatia et al. } \\
\text { (2012) [22] }\end{array}$ & $\begin{array}{l}\text { Hong } \\
\text { Kong }\end{array}$ & Prospective & $\begin{array}{l}2011 \text { Jan- } \\
2011 \text { Jun }\end{array}$ & $\begin{array}{c}46 \\
(23 / 23)\end{array}$ & $\begin{array}{c}58.5 \\
(7-74)\end{array}$ & $\begin{array}{c}56.4 \\
(31 / 55)\end{array}$ & $\begin{array}{l}\text { Squamous cell carcinoma (11), } \\
\text { nasopharyngeal cancer (5), non-Hodgkin's } \\
\text { lymphoma (4), adenocarcinoma of the } \\
\text { lung (5), papillary thyroid cancer (5), poorly } \\
\text { differentiated cancer (1) }\end{array}$ & $\begin{array}{l}\text { Mean: } 30.2 \\
\text { Max: } 45\end{array}$ \\
\hline
\end{tabular}

SSI, supersonic shear imaging; Max, maximum; Min, minimum; SD, standard deviation; na, not reported.

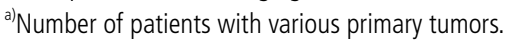


values of the elastic modulus, which were provided in six, nine, four, and three articles, respectively. The detailed characteristics of the 10 included studies are summarized in Table 1.

\section{Risk of Bias}

Two reviewers independently assessed the risk of bias and concerns about applicability based on the QUADAS-2 [8]. The results of the quality assessment using the QUADAS-2 tool are shown in Fig. 2. Overall, the applicability of the included studies was moderate to high, and all the studies satisfied at least four of the seven items.

\section{Diagnostic Accuracy of Different Elastic Modulus Values}

Comparisons of the mean, maximum, minimum, and SD values of the elastic modulus between malignant and benign lymph nodes are displayed in Fig. 3. The pooled differences of all elastic modulus values were statistically significant $(P<0.05)$. However, the heterogenicity between studies could not be ignored.

For the mean elastic modulus, the summary estimates for sensitivity and specificity were $0.720(95 \% \mathrm{Crl}, 0.592$ to 0.824$)$ and 0.877 ( $95 \% \mathrm{Crl}, 0.727$ to 0.969$)$, respectively. The summary estimates for $\mathrm{LR}+$ and $\mathrm{LR}$ - were $5.79(95 \% \mathrm{Crl}, 4.02$ to 12.49$)$ and 0.31 ( $95 \% \mathrm{Crl}, 0.23$ to 0.47 ), respectively. The summary estimates for the diagnostic OR and RD were 16.87 (95\% Crl, 11.17 to 55.39) and $0.58(95 \% \mathrm{Crl}, 0.47$ to 0.72$)$, respectively. The estimated AUC was $0.845(95 \% \mathrm{Crl}, 0.672$ to 0.914$)$. There was no threshold effect according to Spearman correlation analysis $(P=0.064)$. For the maximum elastic modulus, the summary estimates for sensitivity and specificity were $0.809(95 \% \mathrm{Crl}, 0.698$ to 0.899$)$ and $0.816(95 \%$ $\mathrm{Crl}, 0.643$ to 0.924$)$, respectively. The summary estimates for LR+ and LR- were 5.78 (95\% Crl, 3.15 to 11.82) and 0.24 (95\% Crl, 0.13 to 0.34$)$, respectively. The summary estimates for $\mathrm{OR}$ and $\mathrm{RD}$ were $27.67(95 \% \mathrm{Crl}, 11.15$ to 65.75$)$ and $0.67(95 \% \mathrm{Crl}, 0.54$ to 0.76), respectively. The estimated AUC was $0.834(95 \% \mathrm{Crl}, 0.579$ to 0.938$)$. No threshold effect was found ( $P=0.699)$. The details regarding the minimum and SD values of the elastic modulus are shown in Table 2. The corresponding forest and SROC plots are displayed in Figs. 4 and 5, respectively.

\section{Subgroup Analysis}

Data for the mean and maximum values of elastic modulus were divided into subgroups for further analysis. For thyroid cancer the summary estimates for sensitivity and specificity were 0.741 $(0.616-0.834)$ and $0.870(0.583-0.979)$, respectively. The summary estimates for LR+ and LR- were $11.75(95 \% \mathrm{Crl}, 7.55$ to $28.19)$ and $0.30(95 \% \mathrm{Crl}, 0.28$ to 0.40$)$, respectively. The summary

\begin{tabular}{|c|c|c|c|c|c|c|c|c|}
\hline \multirow{2}{*}{\multicolumn{2}{|c|}{$\begin{array}{l}\text { QUADAS-2 } \\
\text { Study ID }\end{array}$}} & \multicolumn{4}{|c|}{ Risk of bias } & \multicolumn{3}{|c|}{ Applicability concerns } \\
\hline & & (1) & (2) & (3) & (4) & (1) & (2) & (3) \\
\hline$\overline{\text { Lo }}$ & 2019 & (;) & (:) & (;) & (;) & (:) & (;) & (:) \\
\hline Kang & 2019 & $?$ & (:) & (:) & (:) & (2) & (:) & (:) \\
\hline You & 2018 & (-) & (:) & (:) & (2) & (:) & (:) & (:) \\
\hline Kim & 2018 & $?$ & (:) & (:) & (:) & (:) & (:) & (:) \\
\hline Chang & 2018 & (:) & (:) & (:) & (:) & (:) & (:) & (:) \\
\hline Chen & 2018 & (:) & (:) & (:) & (:) & (:) & (:) & (:) \\
\hline Desmots & 2016 & (:) & (:) & (:) & (2) & (:) & (2) & (:) \\
\hline Jung & 2015 & $?$ & (:) & (-) & (:) & (:) & (:) & (:) \\
\hline Choi & 2013 & (:) & (:) & (:) & (:) & (:) & (:) & (2) \\
\hline Bhatia & 2012 & (:) & (:) & (:) & (:) & (2) & (2) & (2) \\
\hline
\end{tabular}

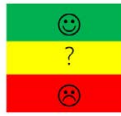

Low risk of bias or concern about applicability Unclear risk of bias or concern about applicability High risk of bias or concern about applicability

(1) Patients selection
(2) Index text
(3) Reference standard
(4)

Fig. 2. Quality assessment of the included studies according to the Quality Assessment of Diagnostic Accuracy Studies-2 (QUADAS-2) criteria.

Table 2. Estimates of diagnostic accuracy from the included studies

\begin{tabular}{|c|c|c|c|c|c|c|c|c|c|}
\hline SSI index & No. of studies ${ }^{a)}$ & $\begin{array}{c}\text { AUC } \\
(95 \% \mathrm{Crl})\end{array}$ & $\begin{array}{c}\text { Sensitivity } \\
(95 \% \text { Crl) }\end{array}$ & $\begin{array}{c}\text { Specificity } \\
(95 \% \text { Crl) }\end{array}$ & $\begin{array}{c}\text { LR+ } \\
(95 \% \mathrm{Crl})\end{array}$ & $\begin{array}{c}\text { LR- } \\
(95 \% \mathrm{Crl})\end{array}$ & $\begin{array}{c}\text { OR } \\
(95 \% \mathrm{Crl})\end{array}$ & $\begin{array}{c}\mathrm{RD} \\
(95 \% \mathrm{Crl})\end{array}$ & $\begin{array}{c}\text { Threshold } \\
\text { effect test } \\
\text { P-value }\end{array}$ \\
\hline Mean & 6 & $\begin{array}{c}0.845 \\
(0.672-0.914)\end{array}$ & $\begin{array}{c}0.720 \\
(0.592-0.824)\end{array}$ & $\begin{array}{c}0.877 \\
(0.727-0.969)\end{array}$ & $\begin{array}{c}5.79 \\
(4.02-12.49)\end{array}$ & $\begin{array}{c}0.31 \\
(0.23-0.47)\end{array}$ & $\begin{array}{c}16.87 \\
(11.17-55.39)\end{array}$ & $\begin{array}{c}0.58 \\
(0.47-0.72)\end{array}$ & 0.064 \\
\hline Max & 9 & $\begin{array}{c}0.834 \\
(0.579-0.938)\end{array}$ & $\begin{array}{c}0.809 \\
(0.698-0.899)\end{array}$ & $\begin{array}{c}0.816 \\
(0.643-0.924)\end{array}$ & $\begin{array}{c}5.78 \\
(3.15-11.82)\end{array}$ & $\begin{array}{c}0.24 \\
(0.13-0.34)\end{array}$ & $\begin{array}{c}27.67 \\
(11.15-65.75)\end{array}$ & $\begin{array}{c}0.67 \\
(0.54-0.76)\end{array}$ & 0.699 \\
\hline Min & 4 & $\begin{array}{c}0.519 \\
(0.101-0.938)\end{array}$ & $\begin{array}{c}0.440 \\
(0.299-0.604)\end{array}$ & $\begin{array}{c}0.923 \\
(0.769-0.991)\end{array}$ & $\begin{array}{c}5.02 \\
(3.11-10.88)\end{array}$ & $\begin{array}{c}0.49 \\
(0.37-0.70)\end{array}$ & $\begin{array}{c}12.13 \\
(4.72-20.49)\end{array}$ & $\begin{array}{c}0.47 \\
(0.27-0.51)\end{array}$ & 0.200 \\
\hline SD & 3 & $\begin{array}{c}0.816 \\
(0.477-0.891)\end{array}$ & $\begin{array}{c}0.782 \\
(0.511-0.921)\end{array}$ & $\begin{array}{c}0.857 \\
(0.727-0.958)\end{array}$ & $\begin{array}{c}4.97 \\
(4.79-5.59)\end{array}$ & $\begin{array}{c}0.29 \\
(0.27-0.29)\end{array}$ & $\begin{array}{c}17.06 \\
(16.72-20.40)\end{array}$ & $\begin{array}{c}0.60 \\
(0.60-0.63)\end{array}$ & $<0.001$ \\
\hline
\end{tabular}

SSI, supersonic shear imaging; AUC, area under the summary receiver operating characteristic curve; Crl, credible interval; LR+, positive likelihood ratio; LR-, negative likelihood ratio; OR, diagnostic odds ratio; RD, risk difference; Max, maximum; Min, minimum; SD, standard deviation.

${ }^{\text {a) }}$ Due to the absence of data, Chan et al's study [17] was omitted from SSI index of Mean and Min. 


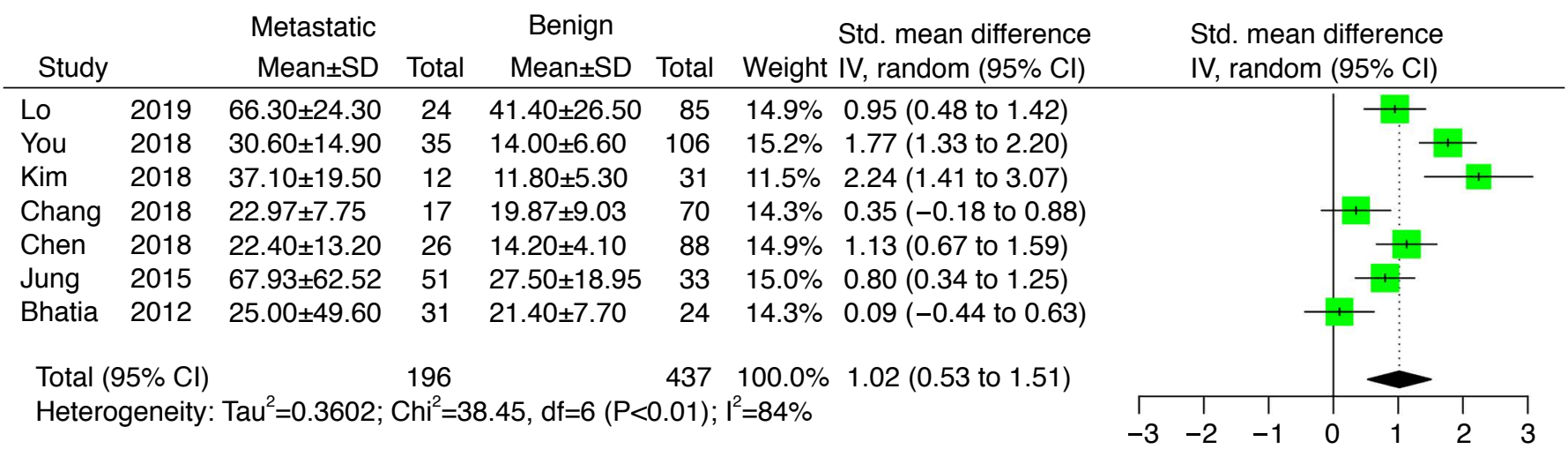

A

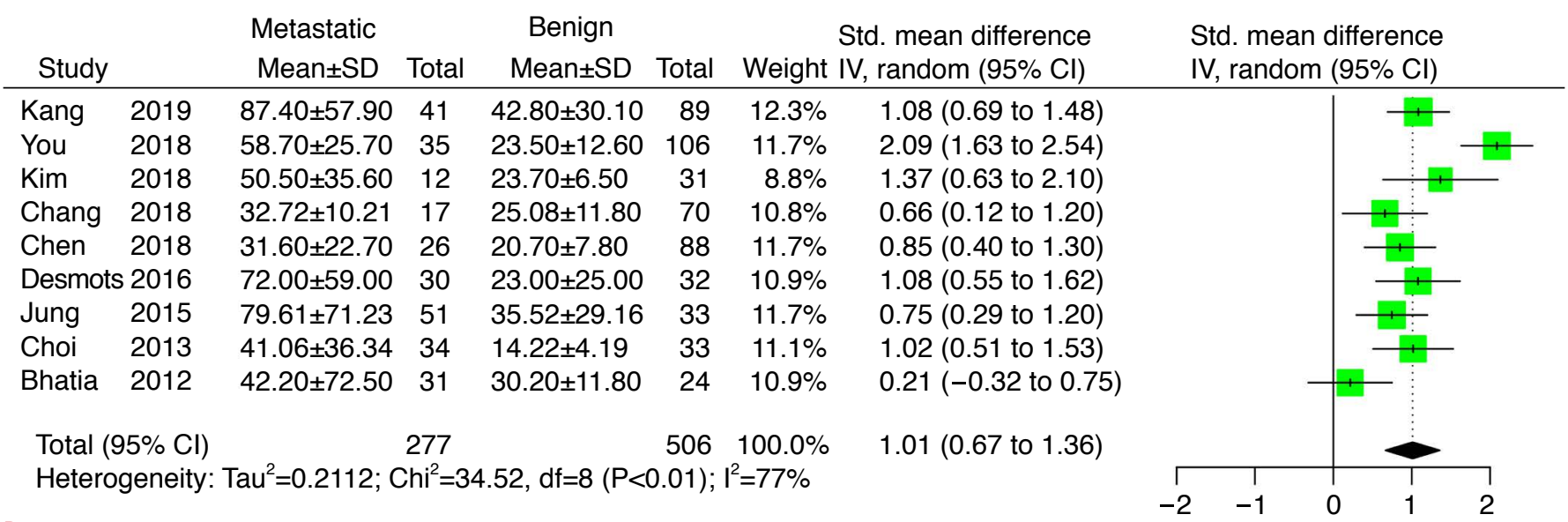
B

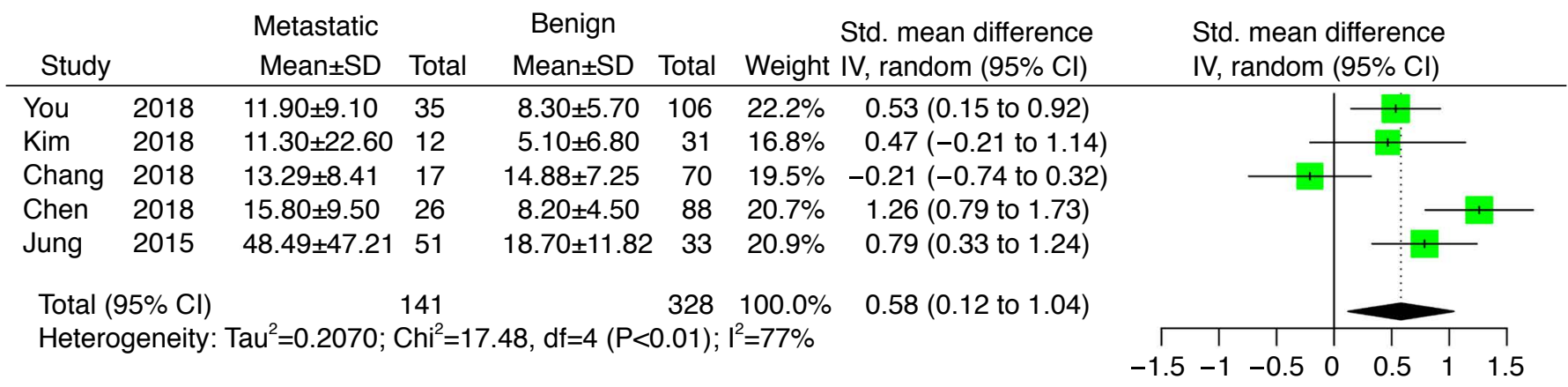
C

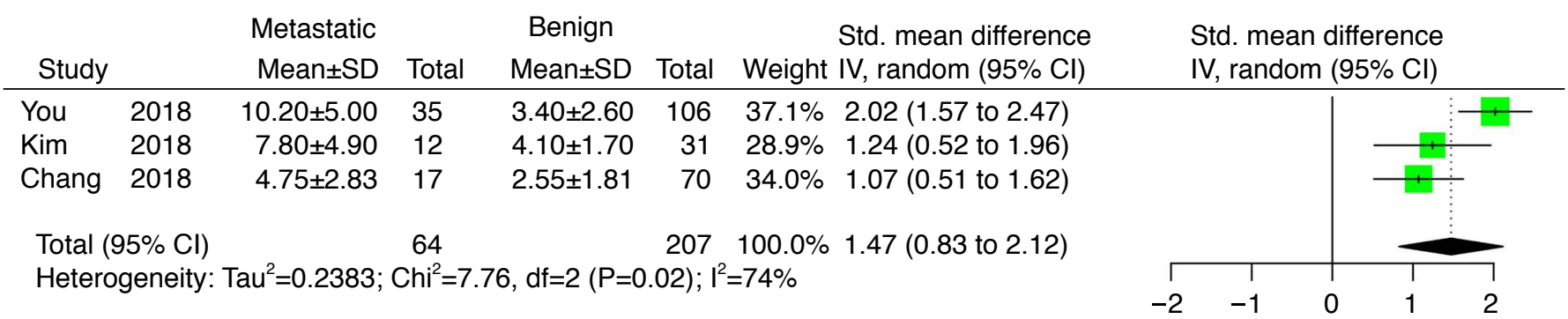

Fig. 3. Mean differences between malignant and benign cervical lymph nodes according to the mean (A), maximum (B), minimum (C), and standard deviation (SD) (D) of the elastic modulus. $\mathrm{Cl}$, confidence interval. 
Study Sensitivity $(95 \% \mathrm{Crl})$

Specificity $(95 \% \mathrm{Crl})$

Lo $20190.811(0.688-0.911)$

You $20180.725(0.605-0.829)$

Kim $2018 \quad 0.657$ (0.458-0.809)

Chen $2018 \quad 0.777(0.661-0.890)$

Jung $2015 \quad 0.777(0.672-0.862)$

$$
\text { 1) }
$$

Bhatia $20120.501(0.323-0.662)$

Estimate $0.720(0.592-0.824)$

A

Sensitivity (95\% Crl)

Study

Kang 20190.691 (0.547-0.808)

You $2018 \quad 0.793(0.659-0.895)$

Kim $2018 \quad 0.802(0.594-0.936)$

Chang 20180.749 (0.549-0.884)

Chen $2018 \quad 0.931(0.817-0.990)$

Desmots 20160.825 (0.691-0.920)

Jung $20150.843(0.737-0.921)$

Choi $2013 \quad 0.871(0.750-0.954)$

Bhatia $20120.552(0.375-0.713)$

Estimate $0.809(0.698-0.899)$

B

Study Sensitivity (95\% Crl)

You $20180.525(0.386-0.675)$

Kim $2018 \quad 0.475$ (0.305-0.667)

Chen 20180.471 (0.334-0.639)

Jung $2015 \quad 0.305(0.185-0.432)$

Estimate $\quad 0.440(0.299-0.604)$

C

Study

Sensitivity (95\% Crl)

You $2018 \quad 0.865$ (0.738-0.956)

Kim $2018 \quad 0.674$ (0.391-0.853)

Chang 20180.753 (0.544-0.883)

Estimate $\quad 0.782(0.511-0.921)$

D

Fig. 4. Estimates of sensitivity and specificity for the mean (A), maximum (B), minimum (C), and standard deviation (D) of the elastic modulus. $\mathrm{Crl}$, credible interval. 
Table 3. Subgroup analysis of the differences in SSI index values between malignant and benign lymph nodes

\begin{tabular}{|c|c|c|c|c|}
\hline Index & Subgroups & No. of studies & $\mathrm{MD}(95 \% \mathrm{Cl})$ & $I^{2}(\%)$ \\
\hline \multirow[t]{8}{*}{ Mean } & Prospective & 5 & 11.24 (3.88 to 18.60$)$ & 83 \\
\hline & Retrospective & 2 & 31.05 (16.66 to 45.44$)$ & 48 \\
\hline & Thyroid cancer & 4 & 19.10 (6.70 to 31.51$)$ & 91 \\
\hline & Other cancer & 3 & 12.70 (0.62 to 24.78) & 74 \\
\hline & Mean age $>50$ & 2 & $15.58(-5.56$ to 36.73$)$ & 76 \\
\hline & Mean age $\leq 50$ & 5 & 15.77 (7.02 to 24.51) & 88 \\
\hline & Malignancy prevalence $>29.7 \%$ & 2 & $21.94(-14.15$ to 58.03$)$ & 88 \\
\hline & Malignancy prevalence $\leq 29.7 \%$ & 5 & 14.43 (6.55 to 22.31$)$ & 87 \\
\hline \multirow[t]{8}{*}{ Max } & Prospective & 6 & 22.57 (10.13 to 35.01$)$ & 87 \\
\hline & Retrospective & 3 & 38.56 (26.89 to 50.23$)$ & 0 \\
\hline & Thyroid cancer & 4 & 27.29 (8.21 to 46.36$)$ & 91 \\
\hline & Other cancer & 5 & 27.76 (12.63 to 42.89) & 78 \\
\hline & Mean age $>50$ & 4 & 28.90 (17.74 to 40.06) & 33 \\
\hline & Mean age $\leq 50$ & 5 & $26.92(11.61$ to 42.23$)$ & 91 \\
\hline & Malignancy prevalence $>29.7 \%$ & 5 & 35.28 (23.12 to 47.45$)$ & 48 \\
\hline & Malignancy prevalence $\leq 29.7 \%$ & 4 & 19.39 (5.08 to 33.69) & 90 \\
\hline
\end{tabular}

SSI, supersonic shear imaging; MD, mean difference; Cl, confidence interval; Max, maximum.

Table 4. Subgroup analysis of the differences in SSI index values between the diagnostic accuracy (sensitivity and specificity)

\begin{tabular}{|c|c|c|c|c|}
\hline Index & Subgroups & No. of studies & Sensitivity (95\% Crl) & Specificity $(95 \% \mathrm{Crl})$ \\
\hline \multirow[t]{8}{*}{ Mean } & Prospective & 4 & $0.727(0.533-0.873)$ & $0.877(0.675-0.983)$ \\
\hline & Retrospective & 2 & $0.739(0.539-0.867)$ & $0.863(0.170-0.998)$ \\
\hline & Thyroid cancer & 3 & $0.741(0.616-0.834)$ & $0.870(0.583-0.979)$ \\
\hline & Other cancer & 3 & $0.721(0.436-0.909)$ & $0.892(0.466-1.000)$ \\
\hline & Mean age $>50$ & 2 & $0.509(0.276-0.774)$ & $0.975(0.892-0.999)$ \\
\hline & Mean age $\leq 50$ & 4 & $0.788(0.703-0.861)$ & $0.673(0.620-0.883)$ \\
\hline & Malignancy prevalence $>29.7 \%$ & 2 & $0.601(0.253-0.876)$ & $0.811(0.343-0.992)$ \\
\hline & Malignancy prevalence $\leq 29.7 \%$ & 4 & $0.781(0.670-0.869)$ & $0.842(0.681-0.950)$ \\
\hline \multirow[t]{8}{*}{ Max } & Prospective & 6 & $0.836(0.647-0.953)$ & $0.838(0.667-0.940)$ \\
\hline & Retrospective & 3 & $0.771(0.608-0.894)$ & $0.753(0.179-0.984)$ \\
\hline & Thyroid cancer & 4 & $0.808(0.711-0.878)$ & $0.814(0.372-0.975)$ \\
\hline & Other cancer & 5 & $0.841(0.561-0.977)$ & $0.807(0.607-0.936)$ \\
\hline & Mean age $>50$ & 4 & $0.734(0.507-0.907)$ & $0.908(0.788-0.981)$ \\
\hline & Mean age $\leq 50$ & 5 & $0.845(0.756-0.922)$ & $0.673(0.402-0.875)$ \\
\hline & Malignancy prevalence $>29.7 \%$ & 5 & $0.762(0.590-0.892)$ & $0.778(0.488-0.951)$ \\
\hline & Malignancy prevalence $\leq 29.7 \%$ & 4 & $0.856(0.657-0.984)$ & $0.814(0.535-0.964)$ \\
\hline
\end{tabular}

SSI, supersonic shear imaging; Crl, credible interval; Max, maximum.

estimates for the OR and RD were 41.68 (95\% $\mathrm{Crl}, 19.22$ to 94.96$)$ and 0.67 (95\% Crl, 0.55 to 0.69 ), respectively. The estimated AUC was 0.768 ( $95 \% \mathrm{Crl}, 0.381$ to 0.859$)$. A threshold effect was found $(\mathrm{P}<0.001)$. For the maximum elastic modulus value, the summary estimates for sensitivity and specificity were $0.808(0.711-0.878)$ and $0.814(0.372-0.975)$, respectively. The summary estimates for LR+ and LR- were 6.14 (95\% Crl, 5.59 to 12.65$)$ and 0.16 (95\% $\mathrm{Crl}, 0.14$ to 0.25$)$, respectively. The summary estimates for the $\mathrm{OR}$ and RD were $39.04(95 \% \mathrm{Crl}, 22.35$ to 87.87$)$ and $0.72(95 \% \mathrm{Crl}$, 0.64 to 0.80$)$, respectively. The estimated AUC was $0.913(95 \%$ 


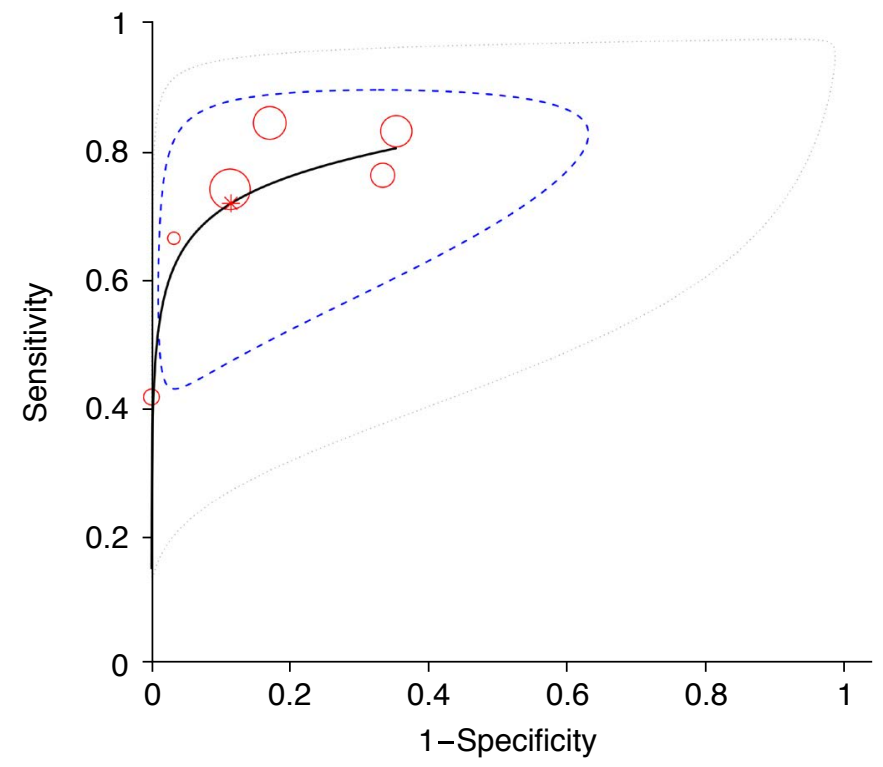

A

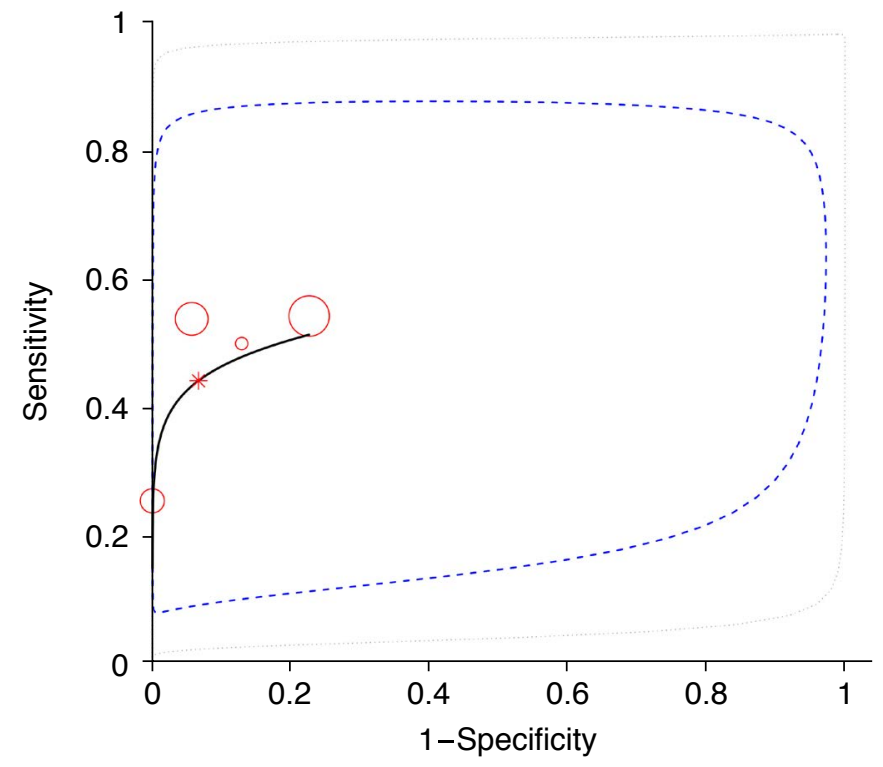

C

Fig. 5. Summary receiver operating characteristic (SROC) curves for the mean (A), maximum (B), minimum (C), and standard deviation (D) of the elastic modulus.

The SROC curve shows individual study posterior-point estimates (the size of each circle is proportional to the sample size for each study). The dashed elliptical boundary represents the $95 \%$ credible region for the summary estimates (closed diamond). The standard (black) and latent-class model analyses based on the conditional dependence model (blue) and the conditional independence model (red) are presented.

$\mathrm{Crl}, 0.889$ to 0.959$)$. No threshold effect was found ( $\mathrm{P}=0.800)$. Additional subgroup analyses regarding study design, other cancers, age, and malignancy prevalence are shown in Tables 3 and 4.

\section{Discussion}

The rising incidence of head and neck cancers requires better

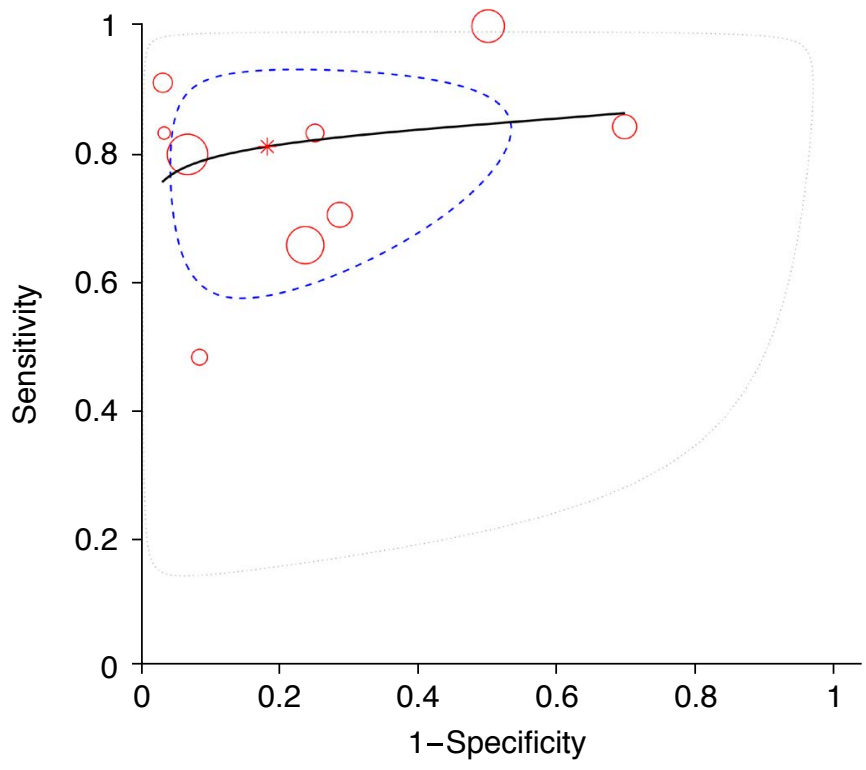

B

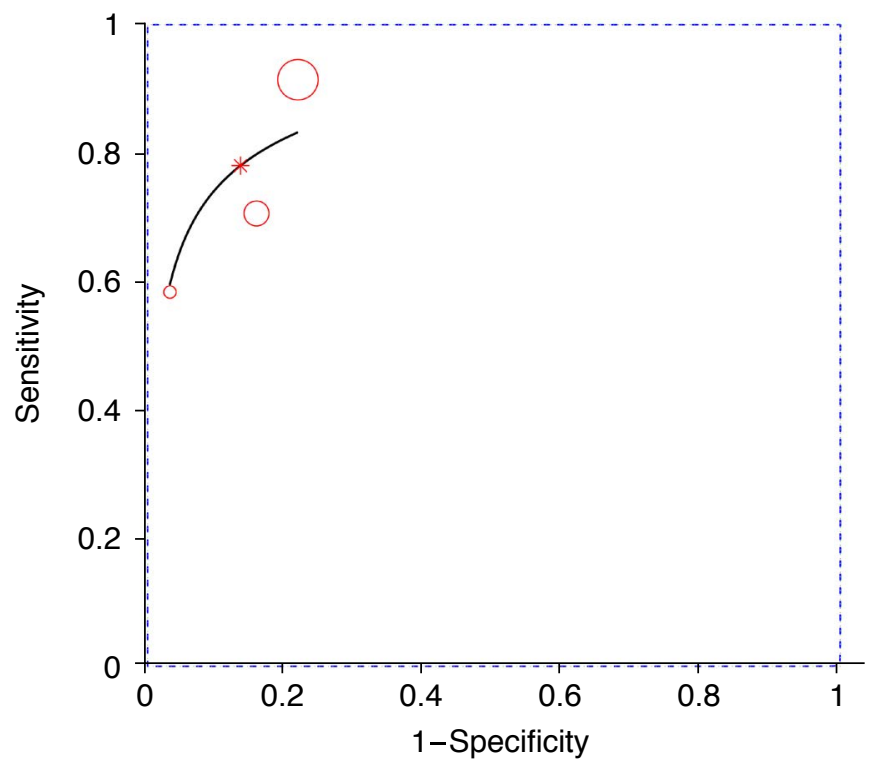

D 
amplify shear waves, which are then tracked throughout the entire window using ultrafast US tracking impulses, while ARFI generates an impulse with a single pushing beam $[5,6]$. As mentioned in a previous meta-analysis, shear wave elastography (including SSI and ARFI) is an acceptable imaging method for diagnosing malignant cervical lymph nodes, with a sensitivity of $0.81(95 \% \mathrm{Cl}, 0.72$ to $0.88)$ and a specificity of $85 \%(95 \% \mathrm{Cl}, 0.70$ to 0.93$)$ [3]. However, the results of SSI elastography from the subgroup analysis showed a sensitivity of $0.80(95 \% \mathrm{Cl}, 0.69$ to 0.91$)$ and a specificity of 0.84 $(95 \% \mathrm{Cl}, 0.67$ to 1.00$)$, although only four original articles were included. In addition, the previous meta-analysis only considered the diagnostic performance of the maximum elastic modulus value.

The current systematic review and meta-analysis first summarized the differences of elastic modulus values (mean, maximum, minimum, and SD) between malignant and benign cervical lymph nodes. Although all elastic modulus values were significantly different, heterogeneity was also obvious. No difference was found between malignant and benign cervical lymph nodes in $28.6 \%$ (2/7) of the articles for the mean elastic modulus, $11.1 \%$ (1/9) for the maximum elastic modulus, $60 \%(3 / 5)$ for the minimum elastic modulus, and $0 \%(0 / 3)$ for the SD of the elastic modulus. These findings indicate that not all parts of malignant lymph nodes were always significantly stiffer than benign lymph nodes, especially for the softest parts. Of course, according to these results, the fact that the maximum elastic modulus of malignant and benign lymph nodes was significantly different should be undisputed, and this finding aligns with the hypothesis that the heterogeneous histology of lymph node metastasis is more accurately reflected by the maximum value for detecting focal cortical metastasis or metastasis where the lymph node is entirely replaced by tumor cells $[19,21]$. It can also be concluded that the variation in the elastic modulus inside the malignant lymph nodes was significantly larger than that in the benign lymph nodes. Bhatia et al. also found that malignant lymph nodes were more likely to have heterogeneous elasticity, even when the nodes appeared uniform on US [22]. Nonetheless, the present results for the MD between malignant and benign cervical lymph nodes regarding each elastic modulus value could be helpful for building a future consensus about cutoff values.

According to the present meta-analysis, the mean elastic modulus showed potential superiority relative to the maximum elastic modulus regarding AUC (0.845 vs. 0.834$)$, but the mean elastic modulus had lower sensitivity (0.720 vs. 0.809$)$ and higher specificity ( 0.877 vs. 0.816 ). However, this difference might have resulted from a different number of included articles and different tumors, and more importantly, no direct evidence was provided. Many previous articles reported comparisons between the mean and maximum elastic modulus values. Bhatia et al. [22] reported the same AUC 0.77 for both mean and maximum elastic modulus values; however, they had distinct advantages regarding sensitivity $(0.484$ vs. 0.419$)$ and specificity $(0.918$ vs. 1.000$)$. Chen et al. [18] found the same trend as in the present study; that is, the AUC of the mean elastic modulus was higher than that of the maximum value $(0.879$ vs. 0.819 ), but the sensitivity and specificity were possibly lower (0.846 vs. 1.000$)$ and higher (0.830 vs. 0.500$)$, respectively. Another article by Jung et al. [20] also reported data supporting the findings of the present study that the mean elastic modulus had a higher AUC (0.748 vs. 0.738$)$, lower sensitivity (0.7647 vs. 0.8431$)$, and higher specificity ( 0.6667 vs. 0.3030 ). Therefore, the choice between different elastic modulus should depend on the situation and actual primary carcinoma. According to the present results, the mean elastic modulus might be more suitable for usual screening while the maximum elastic modulus might be more useful in preoperative evaluations. Meanwhile, the European Federation for Ultrasound in Medicine and Biology guidelines and recommendations suggest that elastography is unlikely to be suitable for the differential diagnosis, but is more likely to be useful for targeting malignant lymph nodes for fine-needle aspiration if multiple lymph nodes are present $[24,25]$. The heterogeneity inside metastatic lymph nodes, represented as the SD of the elastic modulus, was also a good parameter for distinguishing malignant and benign lymph nodes; however, further assessments should be conducted in the future, since only three articles reported this value.

A subgroup analysis showed that the diagnostic performance of SSI for lymph node metastasis from thyroid cancer was not significantly different from its performance for other cancers. The intrinsic curvature of the neck and the superficial location of cervical lymph nodes can cause an inhomogeneous stress distribution in the field of observation, although primary thyroid cancer would have advantages in SSI due to its hardness [14,22]. In addition, the older subgroups showed a trend for lower sensitivity, but higher specificity for both mean and maximum elastic modulus values. Older age is usually a risk factor for early metastasis or recurrence, especially for head and neck cancers [26], and elderly patients were more likely to have been selected for fine-needle biopsy at an early stage, which could have contributed to a higher false-negative rate $[26,27]$. Differences in trends in diagnostic performance according to age could be further investigated by direct comparisons.

Considering that the available studies were all studies with small samples and potential selection bias, a summarized meta-analysis is necessary to elicit results close to the truth. The use of Bayesian theory rather than the $\mathrm{I}^{2}$ method to assess heterogeneity is more precise according to the PRISMA extension for diagnostic test accuracy statement [7]. However, the present meta-analysis has the following limitations. First, only 10 articles were included, and not 
all articles provided data for all four elastic modulus values, which made it impossible to compare the outcomes directly. Secondly, mixed primary carcinomas still contributed to the bias in the present analysis, and it is hoped that further studies will focus on a single tumor type to improve the better validity.

SSI is an acceptable imaging technology for diagnosing malignant cervical lymph nodes and can play a complementary role currently. Both maximum and mean elastic modulus values should be taken into consideration to make a clinical judgment.

ORCID: Yuxuan Qiu: https://orcid.org/0000-0002-5467-6466

\section{Author Contributions}

Conceptualization: Qiu Y, Luo Y. Data acquisition: Qiu Y, Xing Z, Yang Q. Data analysis or interpretation: Qiu Y, Xing Z. Drafting of the manuscript: Qiu Y, Xing Z. Critical revision of the manuscript: Yang Q, Luo Y. Approval of the final version of the manuscript: all authors.

\section{Conflict of Interest}

No potential conflict of interest relevant to this article was reported.

\section{References}

1. Ying L, Hou Y, Zheng HM, Lin X, Xie ZL, Hu YP. Real-time elastography for the differentiation of benign and malignant superficial lymph nodes: a meta-analysis. Eur J Radiol 2012;81:2576-2584.

2. Esen G. Ultrasound of superficial lymph nodes. Eur J Radiol 2006;58:345-359.

3. Suh $\mathrm{CH}$, Choi YJ, Baek JH, Lee JH. The diagnostic performance of shear wave elastography for malignant cervical lymph nodes: a systematic review and meta-analysis. Eur Radiol 2017;27:222-230.

4. Leboulleux S, Girard E, Rose M, Travagli JP, Sabbah N, Caillou B, et al. Ultrasound criteria of malignancy for cervical lymph nodes in patients followed up for differentiated thyroid cancer. J Clin Endocrinol Metab 2007:92:3590-3594.

5. Saadi R, LaRusso S, Vijay K, Goldenberg D. Elastography as a potential modality for screening cervical lymph nodes in patients with papillary thyroid cancer: a review of literature. Ear Nose Throat J 2018;97:31-39.

6. Choi YJ, Lee JH, Baek JH. Ultrasound elastography for evaluation of cervical lymph nodes. Ultrasonography 2015;34:157-164.

7. McInnes MD, Moher D, Thombs BD, McGrath TA, Bossuyt PM; PRISMA-DTA Group, et al. Preferred reporting items for a systematic review and meta-analysis of diagnostic test accuracy studies: the PRISMA-DTA statement. JAMA 2018;319:388-396.

8. Whiting PF, Rutjes AW, Westwood ME, Mallett $S$, Deeks JJ, Reitsma $J B$, et al. QUADAS-2: a revised tool for the quality assessment of diagnostic accuracy studies. Ann Intern Med 2011;155:529-536.

9. Higgins JP, Thompson SG, Deeks JJ, Altman DG. Measuring inconsistency in meta-analyses. BMJ 2003;327:557-560.

10. Rue H, Martino S, Chopin N. Approximate Bayesian inference for latent Gaussian models by using integrated nested Laplace approximations. J R Stat Soc Series B Stat Methodol 2009;71:319392.

11. Guo J, Riebler A, Rue H. Bayesian bivariate meta-analysis of diagnostic test studies with interpretable priors. Stat Med 2017;36:3039-3058.

12. Simpson $D$, Rue $H$, Riebler A, Martins TG, Sorbye S. Penalising model component complexity: a principled, practical approach to constructing priors. Stat Sci 2017;32:1-28.

13. Lo WC, Hsu WL, Wang CT, Cheng PW, Liao LJ. Incorporation of shear wave elastography into a prediction model in the assessment of cervical lymph nodes. PLoS One 2019;14:e0221062.

14. Kang HJ, Seo M, Sohn YM, Yun SJ, Min SY, You MW, et al Comparison of diagnostic performance of B-mode ultrasonography and shear wave elastography in cervical lymph nodes. Ultrasound Q 2019:35:290-296

15. You J, Chen J, Xiang F, Song Y, Khamis S, Lu C, et al. The value of quantitative shear wave elastography in differentiating the cervical lymph nodes in patients with thyroid nodules. J Med Ultrason (2001) 2018:45:251-259.

16. Kim HJ, Choi IH, Jin SY, Park HK, Byun DW, Suh K, et al. Efficacy of shear-wave elastography for detecting postoperative cervical lymph node metastasis in papillary thyroid carcinoma. Int J Endocrinol 2018;2018:9382649.

17. Chang W, Tang L, Lu C, Wu M, Chen M. Shear wave elastography in the evaluation of level VI lymph nodes in papillary thyroid carcinoma: combined with gray-scale ultrasound ex vivo. BMC Cancer 2018;18:1001.

18. Chen BB, Li J, Guan Y, Xiao WW, Zhao C, Lu TX, et al. The value of shear wave elastography in predicting for undiagnosed smal cervical lymph node metastasis in nasopharyngeal carcinoma: a preliminary study. Eur J Radiol 2018;103:19-24.

19. Desmots F, Fakhry N, Mancini J, Reyre A, Vidal V, Jacquier A, et al. Shear wave elastography in head and neck lymph node assessment: image quality and diagnostic impact compared with B-mode and Doppler ultrasonography. Ultrasound Med Biol 2016;42:387-398.

20. Jung WS, Kim JA, Son EJ, Youk JH, Park CS. Shear wave elastography in evaluation of cervical lymph node metastasis of papillary thyroid carcinoma: elasticity index as a prognostic implication. Ann Surg Oncol 2015;22:111-116.

21. Choi YJ, Lee JH, Lim HK, Kim SY, Han MW, Cho KJ, et al. Quantitative shear wave elastography in the evaluation of metastatic cervical lymph nodes. Ultrasound Med Biol 2013;39:935-940.

22. Bhatia KS, Cho CC, Tong CS, Yuen EH, Ahuja AT. Shear wave elasticity imaging of cervical lymph nodes. Ultrasound Med Biol 
2012;38:195-201.

23. Suh CH, Kim SY, Kim KW, Lim YS, Lee SJ, Lee MG, et al. Determination of normal hepatic elasticity by using real-time shearwave elastography. Radiology 2014;271:895-900.

24. Saftoiu A, Gilja OH, Sidhu PS, Dietrich CF, Cantisani V, Amy D, et al. The EFSUMB guidelines and recommendations for the clinical practice of elastography in non-hepatic applications: update 2018. Ultraschall Med 2019;40:425-453.

25. Janssen J, Dietrich CF, Will U, Greiner L. Endosonographic elastography in the diagnosis of mediastinal lymph nodes. Endoscopy 2007;39:952-957.
26. Dong W, Horiuchi K, Tokumitsu H, Sakamoto A, Noguchi E, Ueda $Y$, et al. Time-varying pattern of mortality and recurrence from papillary thyroid cancer: lessons from a long-term follow-up. Thyroid 2019;29:802-808.

27. Sengul D, Sengul I, Van Slycke S. Risk stratification of the thyroid nodule with Bethesda indeterminate cytology, category III, IV, V on the one surgeon-performed US-guided fine-needle aspiration with 27-gauge needle, verified by histopathology of thyroidectomy: the additional value of one surgeon-performed elastography. Acta Chir Belg 2019;119:38-46. 